\title{
Observation of Annealing Twin Nucleation at Triple Lines in Nickel During Grain Growth
}

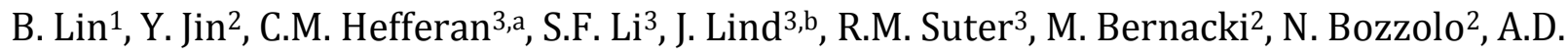
Rollett ${ }^{1}$, G.S. Rohrer ${ }^{1}$

${ }^{1}$ Department of Materials Science and Engineering, Carnegie Mellon University, 5000 Forbes Ave., Pittsburgh, PA 15213-3890, USA

${ }_{2}^{2}$ MINES ParisTech, PSL - Research University, CEMEF - Centre de Mise en Forme des Matériaux, CNRS UMR 7635, CS 10207, 1 rue Claude Daunesse, 06904 Sophia Antipolis cedex, France ${ }^{3}$ Department of Physics, Carnegie Mellon University, 5000 Forbes Ave., Pittsburgh, PA 15213-3890, USA

acurrent affiliation: RJ Lee Group, 350 Hochberg Rd., Monroeville, PA 15146, USA ${ }^{\mathrm{b}}$ current affiliation: Materials Engineering Division, Lawrence Livermore National Laboratory, 7000 East Ave., Livermore, CA 94551, USA

\begin{abstract}
Three-dimensional near-field high-energy x-ray diffraction microscopy has been used to observe the formation of new twinned grains in high purity Ni during annealing at $800{ }^{\circ} \mathrm{C}$. In the fully recrystallized microstructure annealed at $800{ }^{\circ} \mathrm{C}$, twinned grains form along triple lines. Both the grain boundary character and the grain boundary dihedral angles were measured before and after the twin formed. These measurements make it possible to show that although each new twinned grain increases the total grain boundary area, it reduces the total grain boundary energy.
\end{abstract}

Keywords: nickel; twins; grain boundary energy; diffraction 


\section{Introduction}

Annealing twins, separated from parent grains by long straight grain boundaries, are one of the most common and easily recognizable features in face centered cubic (FCC) metals with stacking fault energies less than about $0.15 \mathrm{~J} / \mathrm{m}^{2}$. Examples include metals and alloys such as $\mathrm{Ni}, \mathrm{Cu}, \mathrm{Au}$, brass, and superalloys. Most notable in materials that have undergone recrystallization and grain growth, the twin boundary disorientation is $60^{\circ}$ about a common $<111>$ axis. When the boundary lies in the (111) plane of both crystals, the unique, low energy structure of these (coherent) twins [1, 2] confers improved intergranular properties; this is one of the special boundaries that are exploited in grain boundary engineering [3-5]. Twins in FCC metals have also been implicated in the strengthening of nanostructured $\mathrm{Cu}[6]$, the nucleation of fatigue cracks [7], and the stagnation of grain growth [8]. While methods to increase the twin boundary concentration through thermomechanical processes are known $[3-5,9,10]$, we know much less about the mechanism of annealing twin formation.

The proposed mechanisms for twin formation can be classified into three categories. The first is that twins form when crystals that already have a twin relationship impinge during growth $[11,12]$. The second assumes that a twin forms when growth occurs and a layer of atoms on the (111) plane is misplaced in the twin relationship; further growth on this misplaced layer leads to a twinned crystal [13-16]. The third involves the replacement of higher energy grain boundaries with a combination of a twin boundary and lower energy grain boundaries [17-20]. The available evidence cannot discriminate between these mechanisms for the case of grain growth because twins form within bulk metals that are opaque to visible light and electron beams. This makes it impossible to use standard 
probes to observe the three dimensional structure and crystal orientations before and after the twin has formed. For example, serial sectioning cannot be directly applied to this problem because the sample is destroyed during analysis $[1,21,22]$ and transmission electron microscopy can only visualize the structure within very thin, nearly twodimensional, samples. However, the recent development of near-field high-energy x-ray diffraction microscopy (nf-HEDM) [23-28] and x-ray diffraction contrast tomography [29, 30] enables non-destructive measurements of the shapes and orientations of grains within a bulk sample at sequential stages during annealing.

In this paper, we consider the boundary replacement mechanism for twin formation during normal grain growth. Figure 1 illustrates the most important aspects of the theory [20]. The figure depicts three grains (labeled 1,2, and 3) meeting along a triple line. The grain boundary energies per area $\left(\gamma_{\mathrm{ij}}\right)$, dihedral angles $\left(\theta_{\mathrm{ij}}\right)$, and areas $\left(\mathrm{A}_{\mathrm{ij}}\right)$, are labeled with subscripts that denote the relevant crystals. If a part of grain 2 adjacent to the triple line is replaced by a new grain with the twin disorientation, then a twin boundary is introduced and assumed to be on a (111) plane. The formation of this new grain reduces the energy if the following condition is met [20]:

$$
A_{1 T} \gamma_{1 T}+A_{3 T} \gamma_{3 T}+A_{T} \gamma_{T}<A_{12} \gamma_{12}+A_{23} \gamma_{23}
$$

Given the relatively low energy of the twin $\left(\gamma_{T}=0.06 \mathrm{~J} / \mathrm{m}^{2}\right.$ for $\left.\mathrm{Ni}\right)$ and the significant anisotropy of other grain boundaries [2], there should be many combinations of grains where this condition is met. According to Herring's [31] interfacial equilibrium condition, a 
decrease in the total energy should lead to observable changes in the dihedral angles such that $\theta_{13}^{\prime}<\theta_{13}$ and $\theta_{23}+\theta_{12}<\theta_{1 \mathrm{~T}}+\theta_{3 \mathrm{~T}}$.

Fullman and Fisher [17], and later Murr [20], provided strong albeit indirect evidence for the mechanism depicted in Fig. 1. With previously available techniques, it was simply not possible to observe the same triple junction before and after the event. The repetitive non-destructive three-dimensional structural characterization afforded by nf-HEDM [2328] makes it possible to directly observe the boundary replacement mechanism, if it occurs, and to evaluate the grain boundary crystallography and grain boundary dihedral angles. In this paper, results from high purity Ni annealed at $800{ }^{\circ} \mathrm{C}$ show that twins form at triple lines, as depicted above, and that the process decreases the total interfacial energy.

\section{Methods}

The three-dimensional microstructure of a Ni sample was measured in three different grain growth anneal states using nf-HEDM. The data from each anneal state was compiled as a discrete cubic grid of integers (grain identifiers) that are associated with a set of three Euler angles that describe the orientation of the crystal with respect to the external reference frame. The size of each voxel is $2 \mu \mathrm{m} \times 2 \mu \mathrm{m} \times 4 \mu \mathrm{m}$, in $\mathrm{x}, \mathrm{y}$ and $\mathrm{z}$, respectively. The $x-y$ plane spanned a total of $600 \times 600$ voxels, while the $\mathrm{z}$ dimension was greater than or equal to 70 in each anneal state. The experiment was performed at Argonne National Laboratory's Advanced Photon Source, utilizing the 1-ID beam line to collect orientation diffraction patterns using $65 \mathrm{keV}$ x-rays. The initial sample was a $99.999 \%$ pure $1 \mathrm{~mm}$ diameter nickel wire obtained from Alfa Aesar, which was first homogenized for two hours at $750{ }^{\circ} \mathrm{C}$ to obtain a reasonable grain size for the nf-HEDM method of about $25 \mu \mathrm{m}$. The 
sample was then characterized after three successive annealing steps, all at $800^{\circ} \mathrm{C}$ and in a $3 \% \mathrm{H}_{2} / 97 \% \mathrm{~N}_{2}$ environment to limit oxidation. The three annealing times were $23 \mathrm{~min}$, $30 \mathrm{~min}$, and $25 \mathrm{~min}$. Details of the nf-HEDM experiment and data analysis are given elsewhere [23-28].

The normals to the grain boundary planes, also referred to as the grain boundary orientations, were determined in the following way; a more detailed description can be found in references $[1,22,32]$. First, the traces of each grain boundary on two-dimensional layers were approximated by line segments using the method described by Wright and Larsen [33]. Triple points were identified as three grain boundary traces that shared an end point. Triple points between three crystals with the same orientations on adjacent layers were assumed to be connected by a triple line; the vector product of the triple line and a grain boundary line segment provides the grain boundary normal. The dihedral angles were calculated from the angles between the grain boundary normals.

\section{Results}

An example of the three-dimensional Ni microstructure, interpreted from the nf-HEDM data, is illustrated in Fig. 2. The microstructure of the wire shaped specimen was established by a $2 \mathrm{~h}$ anneal at $750^{\circ} \mathrm{C}$ that recrystallized damage from the wire drawing process. The microstructure contains many twins, which are apparent as long straight boundaries. One particular twinned grain that is visible on two perpendicular sections is indicated by the white arrows. The microstructure was measured in the initial state and after three sequential anneals (each for about $30 \mathrm{~min}$ ) at $800^{\circ} \mathrm{C}$. Three-dimensional representations of the microstructures after the three anneals are illustrated in Fig. S1 and 
show that annealing led to modest but obvious changes in the microstructure. For example, the average grain size (equivalent spherical diameter) increased from $68 \mu \mathrm{m}$ in anneal state 1 to $72 \mu \mathrm{m}$ in anneal state 2 and was roughly constant after that (see Fig. S2). The increase in grain size led to an associated decrease in grain boundary area per volume.

The disorientation distribution measured after each anneal exhibits a significant peak at $60^{\circ}$ (see Fig. S3), and the peak increases by a small amount during annealing. After the initial anneal, boundaries with a $60^{\circ}$ disorientation comprise $25 \%$ of all boundaries (length fraction) and this increases to $29 \%$ after the final anneal. Among these boundaries, coherent twins, the boundaries with a $60^{\circ}$ around [111] and terminated by (111) planes make up $5 \%$ of all boundaries (using a $10^{\circ}$ tolerance on the orientation of the grain boundary plane). Because the thinnest twin plates are not resolved in nf-HEDM, the twin fraction is likely underestimated. Nevertheless, this is the most common boundary in the microstructure and the maximum in the grain boundary plane distribution occurs at the position of the coherent twin, as illustrated in Fig. S4. The increase in the maximum from $590 \mathrm{MRD}$ to $670 \mathrm{MRD}$ indicates an increase in the relative twin area fraction brought about by annealing. This, by itself, is not sufficient to conclude that new twins formed during annealing. The increase in relative area fraction also occurs by the preferential elimination of higher energy grain boundaries as the average grain size increases and the total number of grain boundaries decreases [34].

The detection of new twinned grains requires a detailed comparison of the microstructure at each state. In the overlapping volume of the three anneal states, more than 2000 grains were identified. Using lists of all grains that are completely within the field of view at each anneal step, we identified the subset of grains within this volume that 
did not appear before the anneal and met the following three criteria: First, this disorientation had to be within Brandon's [35] criterion of $60^{\circ}$ around $<111>$ with a neighbor. Second, the grain size had to be greater than 10 voxels. Third, voxels of the new grain had to occur on multiple vertical layers so that the three dimensional shape could be estimated. There were nine grain appearance events that met these criteria. Note that no new grains were detected that did not have the twin disorientation.

One of the grains that appeared is illustrated in Fig. 3. The twinned grain (red) is not visible after the first $23 \mathrm{~min}$ annealing step and then, after an additional $30 \mathrm{~min}$ at $800{ }^{\circ} \mathrm{C}$, appears at a triple line between three grains. When the sample is further annealed for 25 $\min$ at $800^{\circ} \mathrm{C}$, the new grain increases in size from 32 voxels to 117 voxels. It should be noted that according to our conventional ideas about grain growth, small grains and grains with a small number of sides should invariably shrink rather than grow [36, 37]. However, the twinned grains observed here all grew after appearance, suggesting that their growth was energetically favorable. All nine of the new twins that were detected were located at triple lines.

Assuming that local thermal equilibrium is obtained at the triple line, then the Herring [31] condition should describe the relationship between the energies and the geometry of the interface. Under the condition that torque terms (the differentials of the grain boundary energy with respect to changes in the grain boundary plane orientation) are negligible, this reduces to Young's equation:

$$
\frac{\gamma_{12}}{\sin \theta_{12}}=\frac{\gamma_{23}}{\sin \theta_{23}}=\frac{\gamma_{13}}{\sin \theta_{13}}
$$


These quantities are defined in Fig. 1(a). While the assumption of negligible torques is probably not realistic, it has been shown in the past that equation 2 provides a reasonable measure of relative energies $[38,39]$. From equation 2 , if $\gamma_{1 \mathrm{~T}}<\gamma_{12}$, it follows that $\sin \theta_{1 \mathrm{~T}}<$ $\sin \theta_{12}$ and, in the expected domain of $90^{\circ} \leq \theta \leq 180^{\circ}$, this means that $\theta_{1 \mathrm{~T}}>\theta_{12}$. By the same argument, a reduction in boundary energy leads to $\theta_{3 \mathrm{~T}}>\theta_{23}$. In other words, the final dihedral angles after twin formation $\left(\theta_{2 \mathrm{~T}}\right.$ and $\left.\theta_{3 \mathrm{~T}}\right)$ should be greater than the dihedral angles before the twin is formed $\left(\theta_{12}\right.$ and $\left.\theta_{23}\right)$. To test this, the grain boundary planes meeting at the triple junction were determined using previously described methods [1, 22], and the dihedral angles were computed. An uncertainty in the angle, represented by the vertical line through each point, was estimated based on the size of the voxels. The dihedral angles are plotted on Fig. 4(a), which shows that all but one dihedral angle increases after twin insertion, and even that boundary was within the range of estimated uncertainty. However, it should also be recognized that it is not necessary for every single boundary to reduce its energy, only that the sum of the energies $\left(\gamma_{1 T}+\gamma_{3 T}\right)$ after the transition is less than before the transition $\left(\gamma_{12}+\gamma_{23}\right)$, by an amount greater than the energy of the twin boundary. Using Eq. 2, we can write:

$$
\gamma_{12}+\gamma_{23}=\gamma_{13}\left(\frac{\sin \theta_{23}+\sin \theta_{12}}{\sin \theta_{13}}\right)
$$

Because $\gamma_{13}$ is the same before and after twin insertion, we can take the quantities $\left(\sin \theta_{23}+\sin \theta_{12} / \sin \theta_{13}\right)$ and $\left(\sin \theta_{3 \mathrm{~T}}+\sin \theta_{1 \mathrm{~T}} / \sin \theta^{\prime}{ }_{13}\right)$ to represent the sums of the relative grain boundary energies before and after twin insertion and compare them. The 
comparison is illustrated in Fig. 4b, which shows that for all nine triple junctions, twin insertion decreases the energy within the estimated uncertainty.

\section{Discussion}

It should be noted that the new twin-related grains observed here must have started at sizes that were initially below the threshold for detection. The voxel size in the nf-HEDM data is about $2 \mu \mathrm{m} \times 2 \mu \mathrm{m} \times 4 \mu \mathrm{m}$ in the $\mathrm{x}, \mathrm{y}$, and $\mathrm{z}$ directions, respectively. Considering that the twins all grew after detection, we assume that this trajectory was the same since they first formed. As long as the energy criterion in Eq. 1 is satisfied, the growth of the twin is energetically favorable along the two boundaries, provided the boundaries are relatively straight and maintain a similar geometry.

When one considers that there are thousands of triple lines in the specimen, the process observed here is relatively rare. In fact, given reasonable estimates of the grain boundary energies in $\mathrm{Ni},[1,2]$ we can find many other triple lines in this sample where the total energy would be lowered by inserting a twin. One possible explanation for why twins are not inserted at all of the triple lines that have a potential to reduce their energy is that there is a barrier for the initial nucleation of the twinned grain and that this barrier is only occasionally surmounted. The energy balance in Eq. 1 suggests that as long as the criterion is met, energy is always reduced. However, the equation ignores any excess energy associated with the two new triple lines where the twin boundary intersects the preexisting grain boundaries. As long as this energy is finite, then at very small sizes, the formation of the twin grain initially increases the total energy. Therefore, the energies associated with the triple lines are a plausible source of an energy barrier that prevents 
twin formation at all of the triple lines where the energy could be reduced. Assuming that twins form via a nucleation process, one can compare the barriers for the formation of a wedge shaped twin at a triple line (as in Fig. 1(b)) and a hemispherical twin on a flat grain boundary. While the details depend on the exact geometric assumptions, if the triple line energy is assumed to be the same in all cases, then the barrier for nucleation on a grain boundary is at least four times larger than at a grain boundary triple junction. This is consistent with the fact that new twins have only been observed at triple junctions.

There might also be a geometric criterion that must be met for the formation of new twinned grains. The geometry shown in Fig. 1 suggests that the triple line should lie in or near the (111) plane, meaning that the triple line direction should be perpendicular to (and in the zone of) [111]. The directions of the triple lines in crystal space are illustrated Fig. 5. The directions of the triple lines in the reference frame of the twin's parent grain are indicated by the larger circles; eight of nine of these are within $15^{\circ}$ of the (1-11) trace. This indicates that having a triple line approximately perpendicular to the [111] direction may be a moderately strong selection criterion for the nucleation of a twin grain.

Finally, it is also likely that the coupled motion of the grain boundaries and triple lines plays a role in the process. If a triple line is moving in a direction that is reducing the total area of the highest energy boundaries, then any twin that is nucleated may be rapidly consumed. On the other hand, if the triple line is moving in a way that extends the area of these boundaries, the nucleation a twin and the creation of lower energy boundaries would reduce the energy needed for this process. Because of simultaneous changes in the boundary network during annealing, it is difficult to compare the absolute positions of the 
triple line at the different anneal steps with the precision needed to determine the direction of motion.

Because the formation of a new twin at certain triple junctions lowers the total grain boundary energy, it should not be surprising per se. However, because it leads to a temporary and local increase in the total grain boundary area, it contradicts the simple idea that polycrystals reduce their excess interfacial energy by eliminating grain boundaries through grain growth. In fact, it has been shown that the highest energy boundaries are preferentially eliminated during grain growth and this leads to anisotropic grain boundary character distributions [34]. The necessary requirement for this twin formation mechanism is that high energy grain boundaries can be replaced by much lower energy boundaries and the twin; it is assumed that the twin boundary has a much lower energy than the other boundaries. This is true only for certain FCC materials with stacking fault energies that are less than about $0.15 \mathrm{~J} / \mathrm{m}^{2}$. As an example, the twin energy in $\mathrm{Ni}$ is 0.06 $\mathrm{J} / \mathrm{m}^{2}$ or about $6 \%$ of the average grain boundary energy [2]. For comparison, in BCC Fe, the twin energy is $23 \%$ of the average energy [40]; twins in BCC Fe are much less common and this mechanism would not be expected to operate at a measurable rate nor are such long, straight boundaries observed.

We emphasize that the twin formation mechanism described here has been observed at very low capillary driving forces during normal grain growth. It should be noted that during grain growth, the absolute twin content per volume decreases as grains are consumed and twins are annihilated. The process observed here adds some twins, but not at a rate large enough to compensate those lost as grains are eliminated from the microstructure. It is now known that most twins in metals form during recrystallization 
[41], where stored plastic energy provides a driving force for boundary motion that is approximately ten times as large as during grain growth. The extent to which this mechanism is relevant during recrystallization is not clear from the current experiments. However, we note that many twins formed during recrystallization appear as plate-shaped crystals with two parallel twin boundaries. The mechanism described here could not form such a structure, unless the triple junction changed character after the formation of the first boundary. This could happen if grain 1 or 3 was replaced with a new grain during the normal changes in grain connectivity that occur during grain growth, but this was not observed in the present study. As a final note, considering the similarity of grain boundary energy anisotropies in FCC metals [42], we speculate that this same mechanism could happen in isostructural materials.

\section{Conclusion}

The present results demonstrate the utility of nondestructive three-dimensional characterization to measure changes in the grain boundary network during grain growth. The results show that during grain growth in a fully recrystallized microstructure at $800{ }^{\circ} \mathrm{C}$, twins nucleate at triple lines in Ni. The results also show that this process reduces the total grain boundary energy. The selection criteria for the triple junctions that nucleate twins is not currently clear, but they are likely to combine geometric as well as energetic factors.

\section{Acknowledgments}

B.L., A.D.R., and G.S.R. were supported by the Materials World Network of the National Science Foundation (NSF) under Award No. DMR-1107896. Y.J., M.B., and N.B. were 
supported by the French National Research Agency (grant number ANR-11-NS09-001-01).

C.M.H., S.F.L., J.L., R.M.S. were supported by NSF grant DMR-1105173. Use of the Advanced

Photon Source was supported by the US Department of Energy, Office of Science, Office of

Basic Energy Sciences, under contract No. DE-AC02-06CH11357. Research was also

supported in part by the NSF through XSEDE resources provided by Texas Advanced

Computing Center under Award No. DMR-080072.

\section{References Cited}

[1] J. Li, S.J. Dillon, G.S. Rohrer. Relative grain boundary area and energy distributions in nickel, Acta Mater. 57 (2009) 4304-4311.

[2] D.L. Olmsted, S.M. Foiles, E.A. Holm. Survey of computed grain boundary properties in face-centered cubic metals: I. Grain boundary energy, Acta Mater. 57 (2009) 3694-3703.

[3] V. Randle. Twinning-related grain boundary engineering, Acta Mater. 52 (2004) 4067-4081.

[4] V. Randle. Role of grain boundary plane in grain boundary engineering, Mater. Sci. Tech. 26 (2010) 774-780.

[5] T. Watanabe. Grain boundary engineering: historical perspective and future prospects, J. Mater. Science 46 (2011) 4095-4115.

[6] K. Lu, L. Lu, S. Suresh. Strengthening Materials by Engineering Coherent Internal Boundaries at the Nanoscale, Science 324 (2009) 349-352.

[7] C.A. Stein, A. Cerrone, T. Ozturk, S. Lee, P. Kenesei, H. Tucker, R. Pokharel, J. Lind, C. Hefferan, R.M. Suter, A.R. Ingraffea, A.D. Rollett. Fatigue crack initiation, slip localization and twin boundaries in a nickel-based superalloy, Current Opinion in Solid State \& Materials Science 18 (2014) 244-252.

[8] E.A. Holm, S.M. Foiles. How Grain Growth Stops: A Mechanism for Grain-Growth Stagnation in Pure Materials, Science 328 (2010) 1138-1141.

[9] C.S. Kim, Y. Hu, G.S. Rohrer, V. Randle. Five-parameter grain boundary distribution in grain boundary engineered brass, Scripta Mater. 52 (2005) 633-637.

[10] V. Randle, G.S. Rohrer, H.M. Miller, M. Coleman, G.T. Owen. Five-parameter grain boundary distribution of commercially grain boundary engineered nickel and copper, Acta Mater. 56 (2008) 2363-2373.

[11] W.G. Burgers, C.G. Dunn, F. Lionetti, A.J. Shaler, L.D. Jaffe. The effect of orientation difference on grain boundary energies - discussion, Trans. AIMME 185 (1949) 860-862. [12] W.G. Burgers, J.C. Meijs, T.J. Tiedema. Frequency of annealing twins in copper crystals grown by recrystallization, Acta Met. 1 (1953) 75-78.

[13] S. Dash, N. Brown. An investigation of the origin and growth of annealing twins, Acta Met. 11 (1963) 1067-1075.

[14] H. Gleiter. Formation of annealing twins, Acta Met. 17 (1969) 1421-\&. 
[15] S. Mahajan, C.S. Pande, M.A. Imam, B.B. Rath. Formation of annealing twins in f.c.c. crystals, Acta Mater. 45 (1997) 2633-2638.

[16] C.S. Pande, M.A. Imam, B.B. Rath. Study of annealing twins in FCC metals and alloys, Met. Trans. A 21 (1990) 2891-2896.

[17] R.L. Fullman, J.C. Fisher. Formation of annealing twins during grain growth, J. Appl. Phys. 22 (1951) 1350-1355.

[18] P.J. Goodhew. Annealing twin formation by boundary dissociation, Metal Sci. 13 (1979) 108-112.

[19] M.A. Meyers, L.E. Murr. Model for the formation of annealing twins in FCC metals and alloys, Acta Met. 26 (1978) 951-962.

[20] L.E. Murr. Energetics of grain-boundary triple juncitons and corner-twinned junctions - transmission electron microscope studies, J. Appl. Phys. 39 (1968) 5557-5566.

[21] H. Beladi, G.S. Rohrer. The relative grain boundary area and energy distributions in a ferritic steel determined from three-dimensional electron backscatter diffraction maps, Acta Mater. 61 (2013) 1404-1412.

[22] S.J. Dillon, G.S. Rohrer. Characterization of the Grain-Boundary Character and Energy Distributions of Yttria Using Automated Serial Sectioning and EBSD in the FIB, J. Amer.

Ceram. Soc. 92 (2009) 1580-1585.

[23] C.M. Hefferan, J. Lind, S.F. Li, U. Lienert, A.D. Rollett, R.M. Suter. Observation of recovery and recrystallization in high-purity aluminum measured with forward modeling analysis of high-energy diffraction microscopy, Acta Mater. 60 (2012) 4311-4318.

[24] S.F. Li, J. Lind, C.M. Hefferan, R. Pokharel, U. Lienert, A.D. Rollett, R.M. Suter. Threedimensional plastic response in polycrystalline copper via near-field high-energy X-ray diffraction microscopy, J. Appl. Cryst. 45 (2012) 1098-1108.

[25] S.F. Li, R.M. Suter. Adaptive reconstruction method for three-dimensional orientation imaging, J. Appl. Cryst. 46 (2013) 512-524.

[26] J. Lind, S.F. Li, R. Pokharel, U. Lienert, A.D. Rollett, R.M. Suter. Tensile twin nucleation events coupled to neighboring slip observed in three dimensions, Acta Mater. 76 (2014) 213-220.

[27] R.M. Suter, D. Hennessy, C. Xiao, U. Lienert. Forward modeling method for microstructure reconstruction using x-ray diffraction microscopy: Single-crystal verification, Rev. Sci. Instrum. 77 (2006).

[28] L. Wang, J. Lind, H. Phukan, P. Kenesei, J.S. Park, R.M. Suter, A.J. Beaudoin, T.R. Bider. Mechanical twinning and detwinning in pure Ti during loading and unloading - An in situ high-energy X-ray diffraction microscopy study, Scripta Mater. 92 (2014) 35-38.

[29] I.M. McKenna, S.O. Poulsen, E.M. Lauridsen, W. Ludwig, P.W. Voorhees. Grain growth in four dimensions: A comparison between simulation and experiment, Acta Mater. 78 (2014) 125-134.

[30] M. Syha, W. Rheinheimer, M. Baurer, E.M. Lauridsen, W. Ludwig, D. Weygand, P. Gumbsch. Three-dimensional grain structure of sintered bulk strontium titanate from X-ray diffraction contrast tomography, Scripta Mater. 66 (2012) 1-4.

[31] C. Herring. Surface tension as a motivation for sintering. in: Kingston WE, (Ed.). The physics of powder metallurgy. McGraw-Hill New York, 1951. pp. 143-179.

[32] G.S. Rohrer, J. Li, S. Lee, A.D. Rollett, M. Groeber, M.D. Uchic. Deriving grain boundary character distributions and relative grain boundary energies from three-dimensional EBSD data, Mater. Sci. Tech. 26 (2010) 661-669. 
[33] S.I. Wright, R.J. Larsen. Extracting twins from orientation imaging microscopy scan data, J. Micro. 205 (2002) 245-252.

[34] S.J. Dillon, G.S. Rohrer. Mechanism for the development of anisotropic grain boundary character distributions during normal grain growth, Acta Mater. 57 (2009) 1-7. [35] D.G. Brandon. Structure of high-angle grain boundaries, Acta Met. 14 (1966) 14791484.

[36] W.W. Mullins. 2-dimensional motion of idealized grain boundaries, J. Appl. Phys. 27 (1956) 900-904.

[37] D.J. Rowenhorst, A.C. Lewis, G. Spanos. Three-dimensional analysis of grain topology and interface curvature in a beta-titanium alloy, Acta Mater. 58 (2010) 5511-5519.

[38] G.S. Rohrer. Grain boundary energy anisotropy: a review, J. Mater. Sci. 46 (2011) 5881-5895.

[39] C.S. Smith. Grains, Phases, and Interfaces - and interpretation of Microstruture, Trans. AIMME 175 (1948) 15-51.

[40] S. Ratanaphan, D. Olmsted, V.V. Bulatov, E.A. Holm, A. Rollett, G.S. Rohrer. Grain boundary energies in body-centered cubic materials, Acta Mater. 88 (2015) 346-354. [41] Y. Jin, B. Lin, M. Bernacki, G.S. Rohrer, A.D. Rollett, N. Bozzolo. Annealing twin development during recrystallization and grain growth in pure nickel, Mater. Sci. Eng. A 597 (2014) 295-303.

[42] E.A. Holm, D.L. Olmsted, S.M. Foiles. Comparing grain boundary energies in facecentered cubic metals: Al, Au, Cu and Ni, Scripta Mater. 63 (2010) 905-908. 


\section{Figure Captions}

Figure 1. A schematic illustration of a triple junction before (a) and after (b) a new grain, twinned with respect to grain 2 and labeled 'twin', is inserted. Three grain boundaries separating grains 1,2 , and 3 , meet along the triple line labeled $t$. The schematic defines all of the parameters referred to throughout this paper. Adapted from Murr, 1968 [20].

Figure 2. Visual representation of the Ni microstructure measured by nf-HEDM. The sample was a $1 \mathrm{~mm}$ diameter wire; a $90^{\circ}$ wedge has been made invisible to illustrate the internal structure. The grains are colored randomly so that discrete changes in color correspond to grain boundaries. The white arrows point to two traces of a twin boundary on perpendicular sections.

Figure 3. Illustration of three grains that meet at a triple line where a new twinned grain appears. (a-c) three-dimensional representations of the three grains. The peach (1) and olive (3) colored grains are semi-transparent to show the blue (2) grain in the rear and their line of intersection. The viewing direction is close to the plane of the boundary between the peach and olive colored grains. (a) Microstructure after $23 \mathrm{~min}$ at $800{ }^{\circ} \mathrm{C}$, (b) after an additional $30 \mathrm{~min}$ at $800{ }^{\circ} \mathrm{C}$, and (c) after an additional $25 \mathrm{~min}$ at $800{ }^{\circ} \mathrm{C}$. The red grain that appears in (b) is at the line of intersection between the three grains and is twin related to the blue grain. (d-e) Plane sections of the same three grains through the triple line where the twin forms.

Figure 4. (a) The final dihedral angles $\left(\theta_{2 \mathrm{~T}}\right.$ and $\left.\theta_{3 \text { T }}\right)$ after twin formation (two per new grain) plotted against the angles before the twin formed $\left(\theta_{12}\right.$ and $\left.\theta_{23}\right)$. (b) The relative grain boundary energy after each twin formation event is compared to the relative energy before twin formation. In both plots, the dashed line marks the positions where the two quantities are equal. In (a) the vertical line through each data point marks the estimated uncertainty and in (b) it marks the uncertainty that would maximize the final energy; the uncertainty in the other direction is similar.

Figure 5. The directions of triple lines, in crystal space, plotted in stereographic projection, for the nine triple lines where a twin was detected. The directions of each triple line in the reference frame of each of the three crystals are plotted; the larger circles are the direction in the parent grain. The dashed line corresponds to the (1-11) trace. 

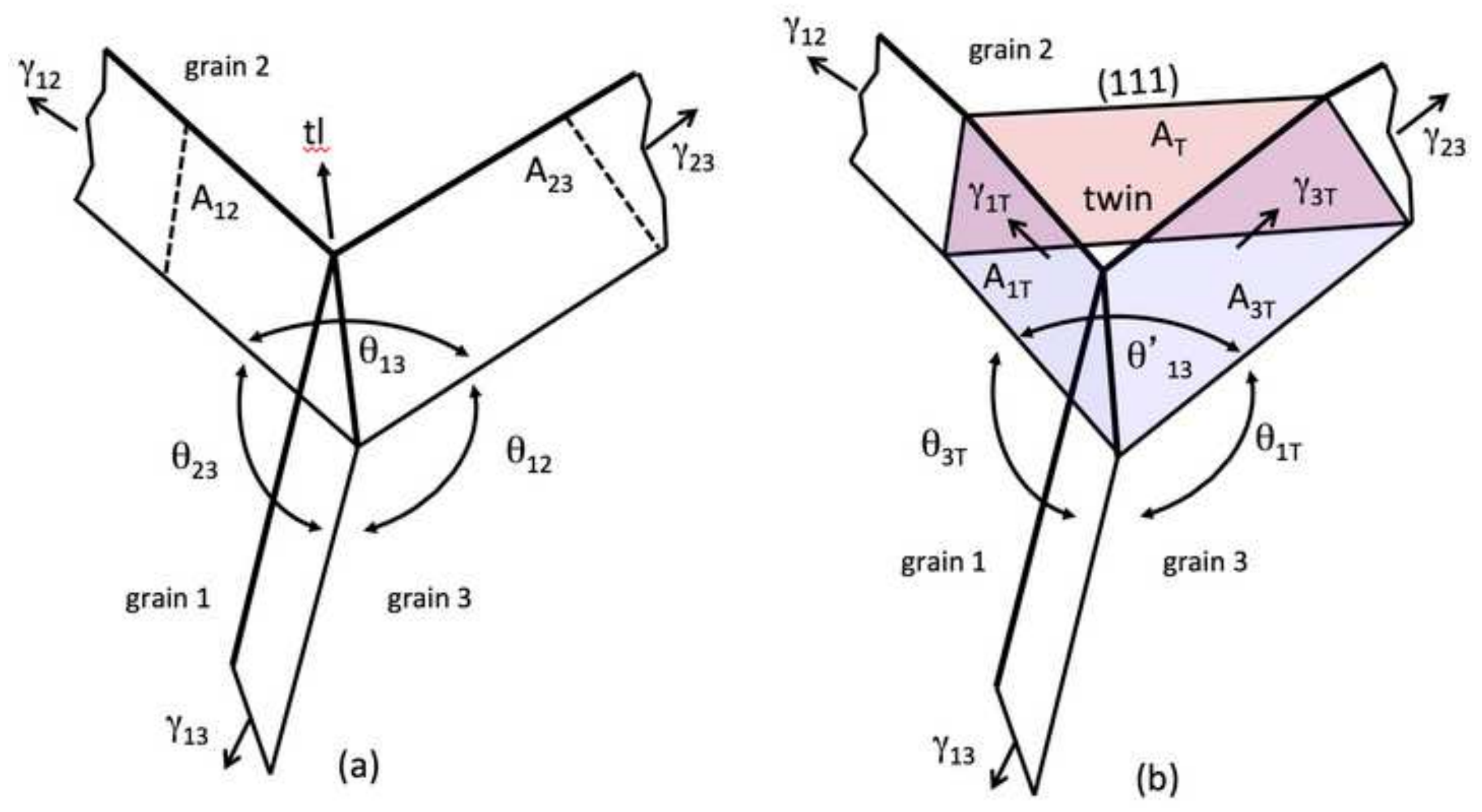

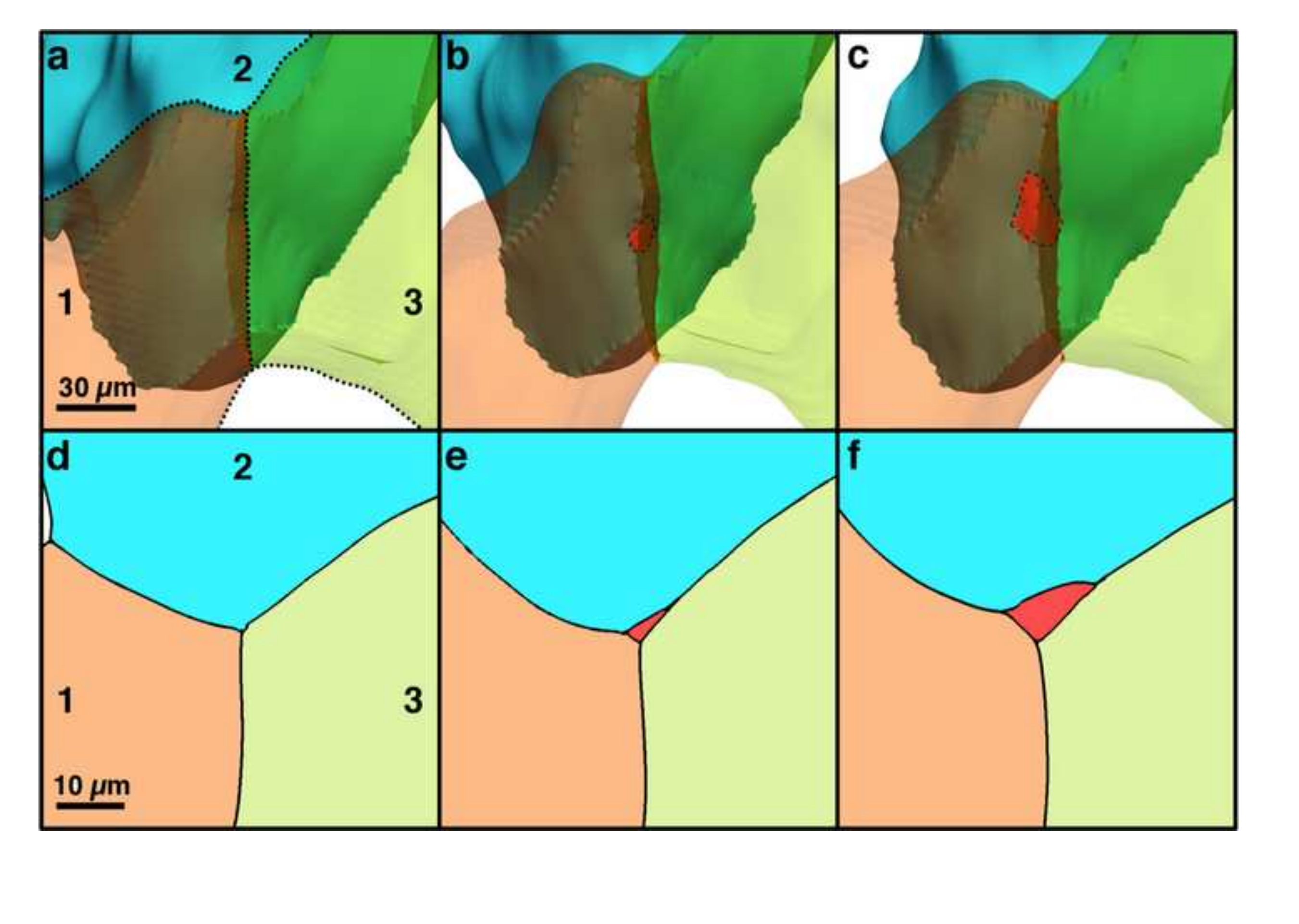

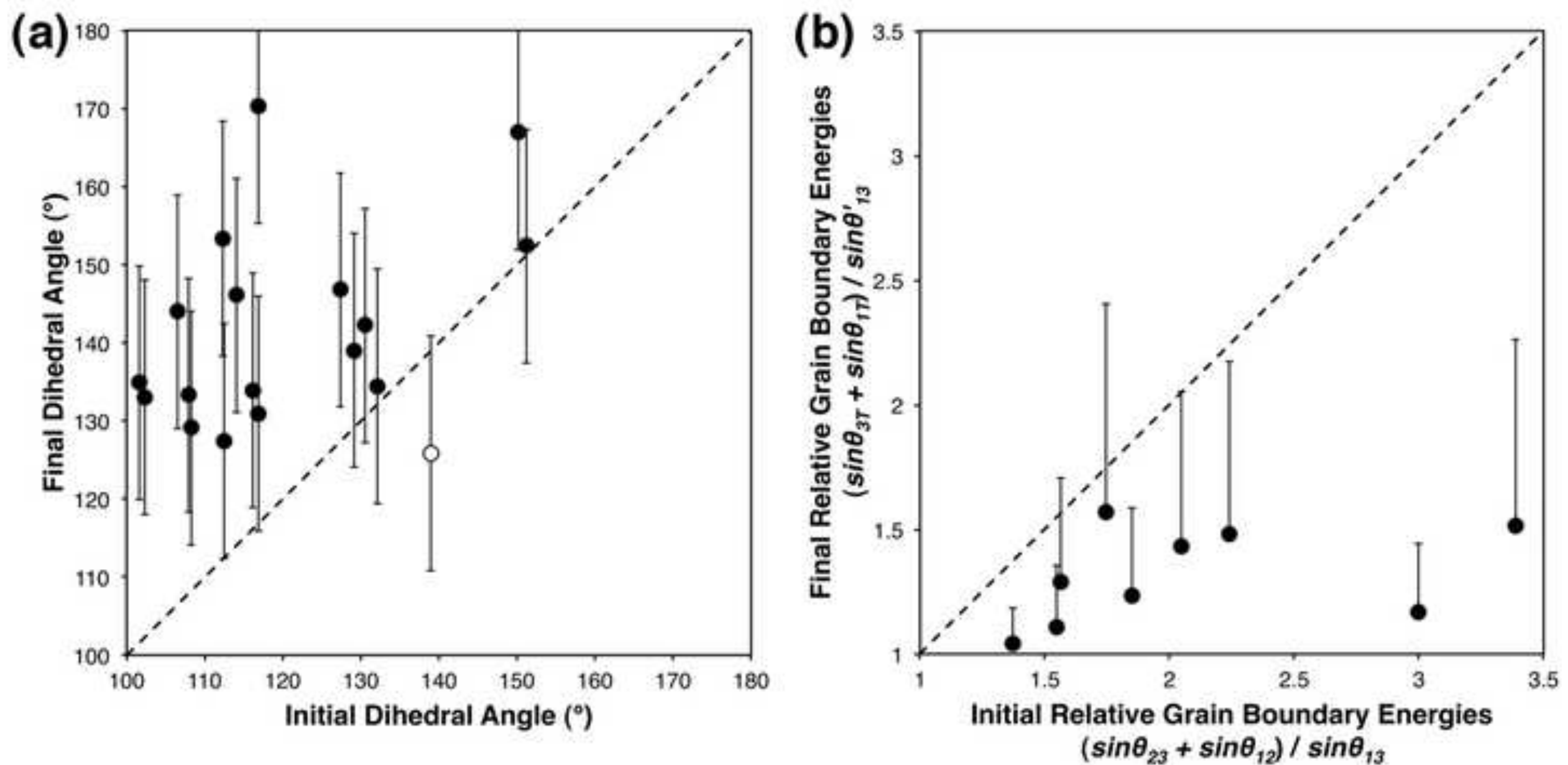


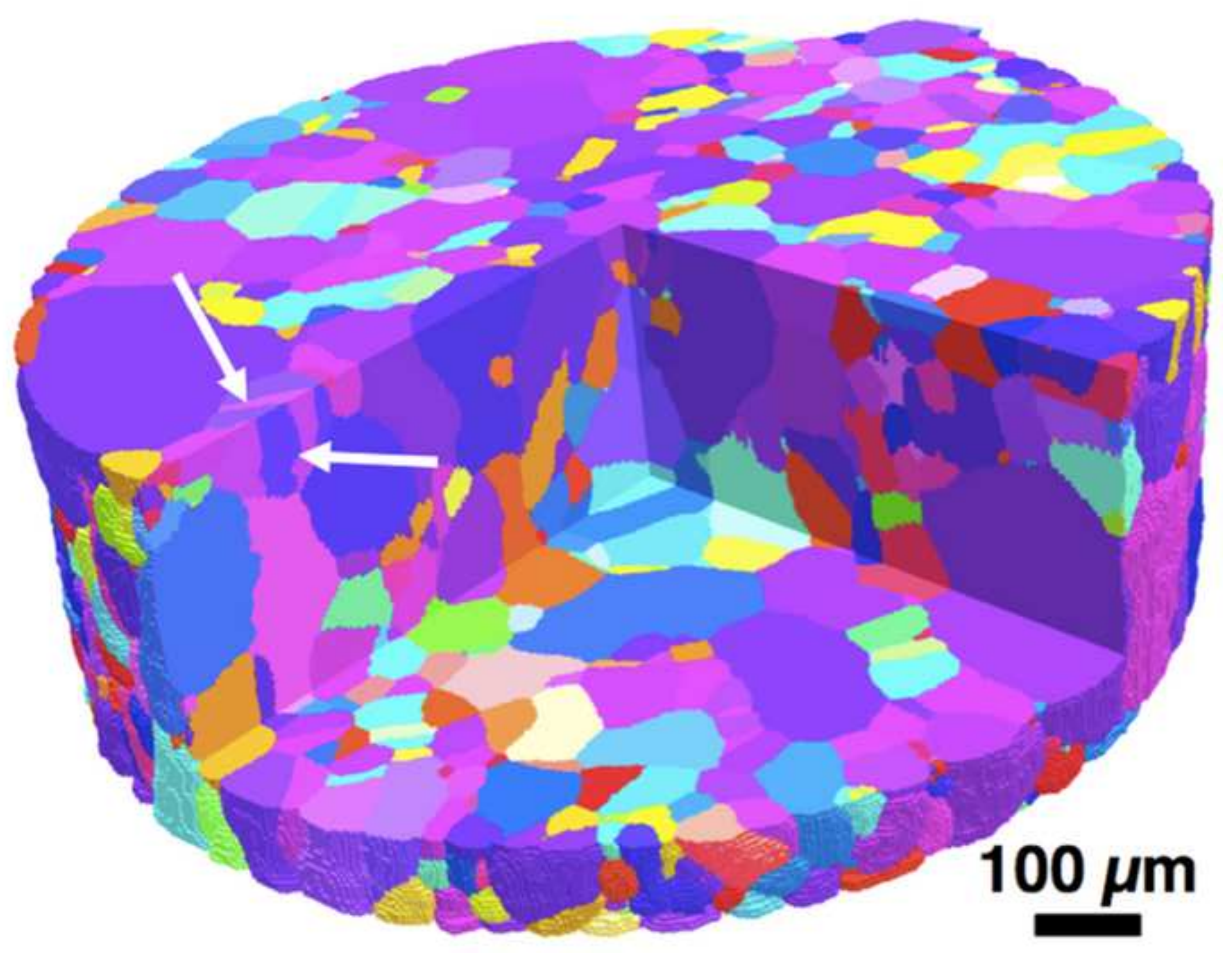

